\title{
A COMBINATION OF THE SCALED BOUNDARY FINITE ELEMENT METHOD WITH THE MORTAR METHOD
}

\author{
Jannis Bulling ${ }^{1}$ AND Hauke Gravenkamp ${ }^{2}$ \\ ${ }^{1}$ Bundesanstalt für Materialforschung und -prüfung (BAM) \\ Unter den Eichen 87, 12205 Berlin, Germany \\ jannis.bulling@bam.de \\ ${ }^{2}$ University of Duisburg-Essen \\ Universitätsstraße 15, 45141 Essen, Germany \\ hauke.gravenkamp@uni-due.de
}

Key words: Ultrasound, Scaled Boundary Finite Element Method, Mortar Method, Domain Decomposition

\begin{abstract}
Connecting different domains is one possibility to increase the performance of a numerical solution method. The Mortar Method is one of the well-established methods for this task. In this contribution, we focus on the solution of the elastodynamic wave equation by means of the scaled boundary finite element method and demonstrate that it is straightforward to connect different polygonal meshes by employing the Mortar Method in two dimensions. Examples show the stability for higher-order shape functions when performing h-refinement or p-refinement.
\end{abstract}

\section{INTRODUCTION}

The simulation of ultrasonic waves in a linearly elastic body can be computationally intensive. The reason is the relatively short wavelength compared to the body's dimensions at high frequencies. One possible approach to counteract the high computational costs is to decompose the domain into smaller parts. Each subdomain may be assigned its own efficient simulation method, and the subdomains can be solved in parallel. The Mortar Method is a well-established approach for coupling such subdomains [1, 2], allowing the combination of multiple solution methods within the same model. For elastodynamic waves, a few of many examples can be found in $[3,4,5]$. In the simplest case, different meshes can be connected.

In recent years, discretizations by polygonal elements have been of interest as they show the same flexibility as triangular elements for meshing while offering additional benefits. These benefits can be a smaller number of degrees of freedom to obtain the same accuracy. They also often show a better stability in case of distorted elements [6, 7]. Approaches to generating polygonal elements are the Voronoi Cell Finite Element Method [8], the Virtual Element Method [9] and finite element formulations based on the generalized barycentric coordinates [10,11, 12], to name a few. The Virtual Element Method is based on shape functions which solve the differential equation, but are not directly computed; this leads to the name 'virtual.' The Voronoi Cell Finite Element Method, on the other hand, uses explicit stress functions to calculate the polygons' stiffness matrix. 
Another approach to discretizing the emerging polygonal elements is the Scaled Boundary Finite Element Method [13, 14]. This semi-analytical method has many attractive properties, some of which are listed subsequently. A variety of material distributions, including anisotropic materials, can be considered. High-order shape functions can be used for optimal convergence properties [14, 15]. The approach treats singularities at crack tips and corners analytically $[13,16]$. Especially in the frequency domain, the Scaled Boundary Finite Element Method reduces the approximation dimension because only degrees of freedom associated with the boundary of a polygonal element are required. Those desirable properties make the method particularly suitable for simulating the dynamic response in bodies with cracks, as is essential for many non-destructive testing and structural health monitoring applications $[17,18]$.

We present in this contribution a combination of the Scaled Boundary Finite Element Method with the Mortar Method in two dimensions. The second section gives a theoretical overview of both approaches. Subsequently, numerical examples demonstrate the stability of the combination for the polygonal boundary of the elements. The numerical models increase in complexity and are compared to results computed on non-divided domains by means of the Spectral Element Method (SEM) [15] .

\section{Theory}

This article considers the linear elastic wave equation on multiple subdomains. For the sake of simplicity, only two subdomains are used in the notation. The subdomains $\Omega_{i}, i \in\{1,2\}$, are non-overlapping and have a common internal boundary $\Gamma_{12}$. In general, however, the approach can be extended to any number of subdomains. The strong form of the problem is
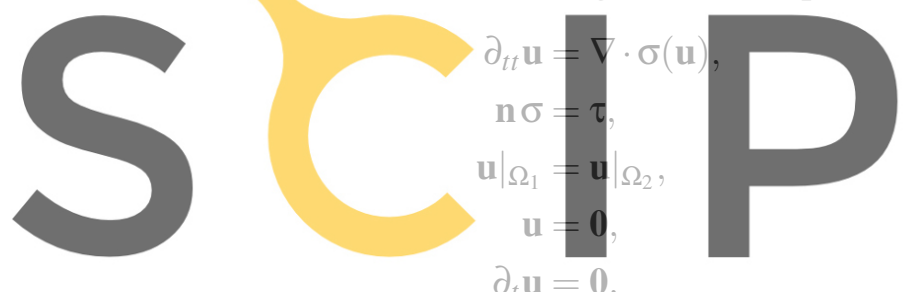
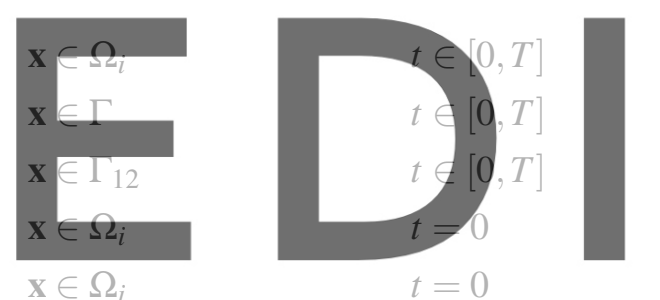

$\mathbf{X} \in \Omega_{i}$

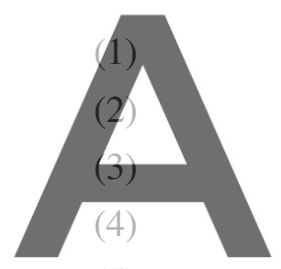

(5)

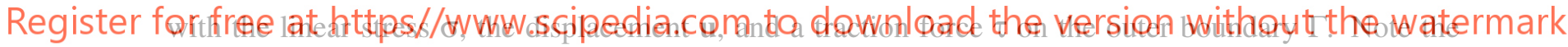
continuity condition on the internal boundary $\Gamma_{12}$ in Equation (3). Figure 1 shows two polygonal meshes, which are coloured light and dark green indicating the subdomains. The common boundary is marked with a black line.

The Scaled Boundary Finite Element Method is deployed to approximate the linear elastic wave equation in each subdomain. For the approximation, the subdomains must be subdivided into polygons, which we call S-elements. In this article, the following approach is used to derive a polygonal mesh and its stiffness matrix:

- Start with a triangulation of the subdomain. For this paper, the triangulations are generated by 'gmsh' [19].

- Use the dual mesh of this triangulation to obtain a polygonal mesh. The dual mesh is constructed by defining each triangle's midpoints and connecting these midpoints of adjacent triangles. There are special steps to define the polygons next to the boundary. This approach is similar to [20].

- For each polygon, the local stiffness matrix is computed and assembled into the global stiffness matrix analogously to the Finite Element Method. 
a)

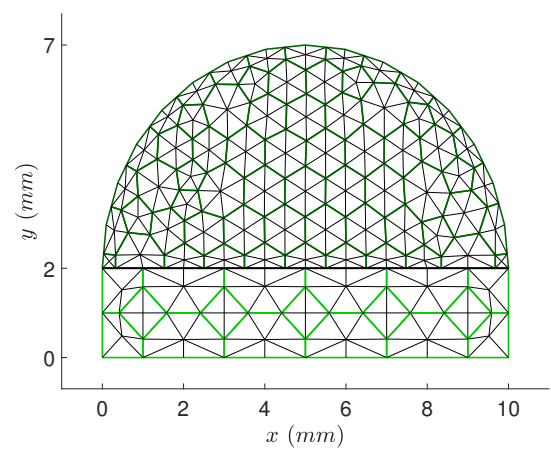

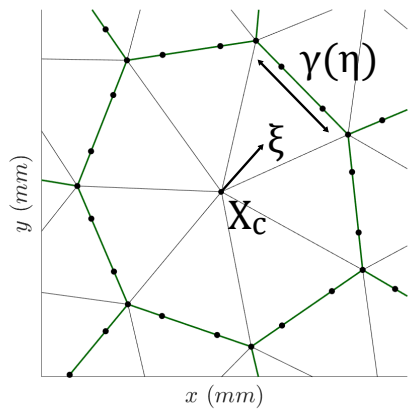

b)

Figure 1: a) Example mesh and b) Close up of a polygon

In the following paragraphs, we will summarize the crucial points for the derivation of the local stiffness matrices of each polygon.

The polygon's stiffness matrix is computed with the help of a particular coordinate system, called the scaled boundary coordinate system (see Figure $1 b)$. Let $\boldsymbol{\gamma}(\eta)$ be a boundary parameterization given by straight line segments, where $\eta$ is a local coordinate for each element. Choose a point $\mathbf{x}_{c}$ that "sees" the entire boundary. Then, the scaled boundary coordinates are defined as

$$
\mathbf{x}=\xi\left(\boldsymbol{\gamma}(\eta)-\mathbf{x}_{C}\right)+\mathbf{x}_{C},
$$

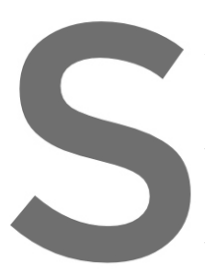

where the point $\mathbf{x}_{C}$ is called the several types of shape functi tral shape functions of art straight lines for the boun tions $\mathrm{N}^{1 / d}$ for $\mathrm{p}=3$. T
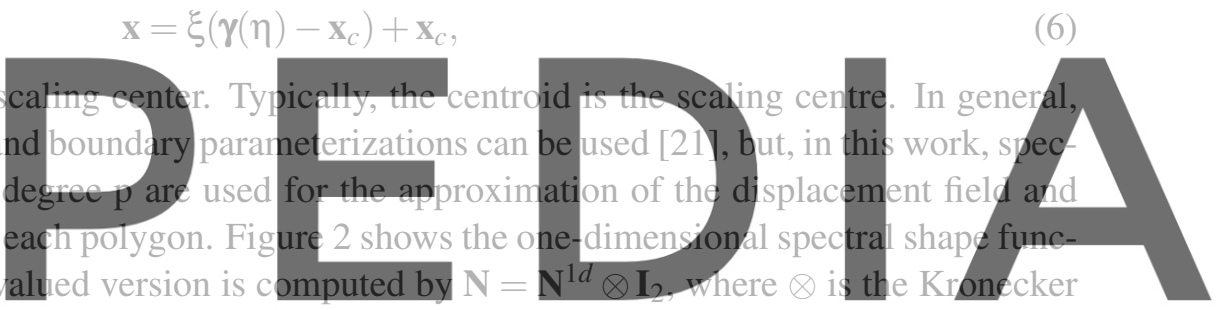

tensor product, and $\mathbb{I}_{2}$ is the identity matrix of size two.

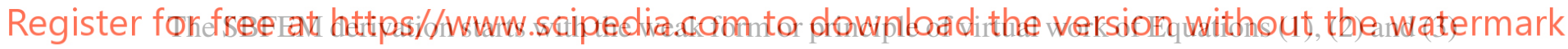
in the frequency domain, that is,

$$
-\int \delta \mathbf{u} \cdot \omega^{2} \mathbf{u} \mathrm{d} \Omega_{i}=-\int \nabla \delta \mathbf{u} \cdot \sigma(\mathbf{u}) \mathrm{d} \Omega_{i}+\int \delta \mathbf{u} \cdot \tau \mathrm{d} \Gamma+I_{12}
$$

with the angular frequency $\omega$. The term $I_{12}$ enforces the continuity condition, but does not influence the derivation of the polygons and will be covered in Equation (22). The finite elements can be used to re-write the weak form in Equation (7) as an ordinary differential equation in the scaled boundary coordinate system, called the SBFEM-equation for the nodal displacement in a polygonal S-element:

$$
\xi^{2} \mathbf{E}_{0} \partial_{\xi \xi} \mathbf{u}(\xi)+\xi\left(\mathbf{E}_{0}+\mathbf{E}_{1}^{\top}-\mathbf{E}_{1}\right) \partial_{\xi} \mathbf{u}(\xi)-\mathbf{E}_{2} \mathbf{u}(\xi)+\xi^{2} \omega^{2} \mathbf{M}_{0} \mathbf{u}(\xi)=\mathbf{0}
$$

Note that $\mathbf{u}(\xi)$ is still a function of $\xi$. The matrices $\mathbf{E}_{i}$ are associated with the stress, and $\mathbf{M}_{0}$ is the boundary mass matrix,

$$
\begin{array}{rlrl}
\mathbf{M}_{0}=\int \mathbf{N}(\eta)^{\top} \rho(\eta) \mathbf{N}(\eta)|j(\eta)| \mathrm{d} \eta, & & \mathbf{E}_{0}=\int \mathbf{B}_{1}(\eta)^{\top} \mathcal{D}(\eta) \mathbf{B}_{1}(\eta)|j(\eta)| \mathrm{d} \eta, \\
\mathbf{E}_{1}=\int \mathbf{B}_{2}(\eta)^{\top} \mathcal{D}(\eta) \mathbf{B}_{1}(\eta)|j(\eta)| \mathrm{d} \eta, & \mathbf{E}_{2}=\int \mathbf{B}_{2}(\eta)^{\top} \mathcal{D}(\eta) \mathbf{B}_{2}(\eta)|j(\eta)| \mathrm{d} \eta,
\end{array}
$$


a)

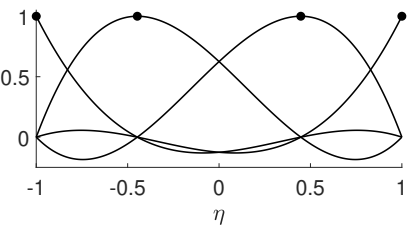

b)

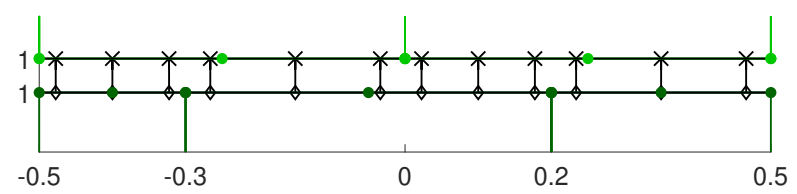

Figure 2: a) Spectral shape functions and b) Quadrature close up

where $\mathbf{B}_{i}$ are matrices that involve the shape functions and their derivatives with respect to $\eta, \mathcal{D}$ is the linear elastic tensor in Voigt notation, and $j(\eta)$ denotes the $\xi$-independent part of the Jacobi-determinant. Every column of these matrices corresponds to one shape function - similar to the FEM. A detailed derivation can be found in [13].

Assume that the inner nodal forces $\mathbf{q}$ can be expressed by a linear stiffness matrix $\mathbf{S}(\xi, \omega)$, i.e.,

$$
\begin{aligned}
\mathrm{q} & =\left(\xi \mathbb{E}_{0} \partial_{\xi}+\mathbb{E}_{1}^{\top}\right) \mathbf{u}(\xi) \\
& =\mathrm{S}(\xi, \omega) \mathbf{u}(\xi) .
\end{aligned}
$$

Considerations regarding the scalability of the wave equation for linear elastic problems lead to the assumption that the stiffness matrix is a function of a single quantity $\chi=(i \omega \xi)^{2}$, i.e., $\mathbf{S}(\xi, \omega)=\mathbf{S}(\chi)$. After a few transformations, one obtains the following matrix differential equation by considering an arbitrary displacement $[1$
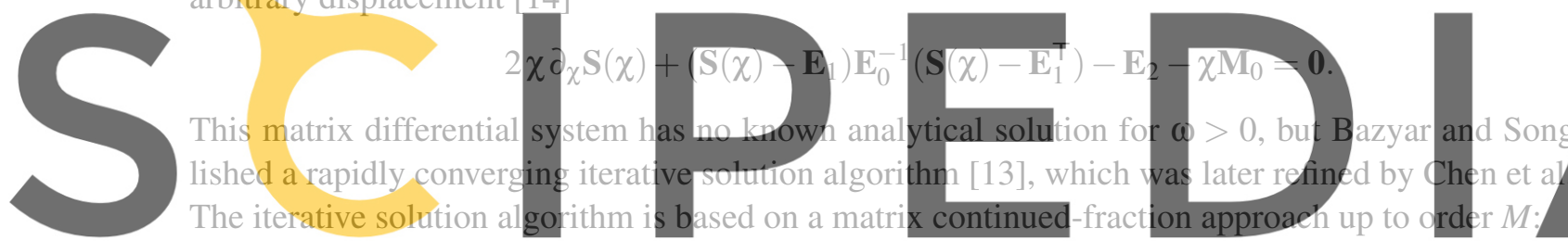

$$
\mathbf{S}(\chi)=\mathbb{K}+\chi \mathbf{M}-\chi^{2} \mathbf{X}^{(1)}\left(\mathbf{S}^{(1)}(\chi)\right)^{-1}\left(\mathbf{X}^{(1)}\right)^{\top}
$$

(14)

Register for free at https//wwww.scip,edia $c_{0, m}$ to download the versipn without the watermark

$$
\mathrm{S}^{(M)}(\chi)=\mathrm{S}_{0}^{(M)}+\chi \mathrm{S}_{1}^{(M)}
$$

where $\mathbf{K}, \mathbf{M}$ are low-order approximations of the static stiffness and mass matrix, respectively. The construction of the higher-order matrices $\mathbf{S}_{0}^{(m)}, \mathbf{S}_{1}^{(m)}$, the pre-conditioner matrix $\mathbf{X}^{(m)}$, as well as $\mathbf{K}, \mathbf{M}$ can be found in [14]. At the boundary $(\xi=1)$, the inner nodal forces have to coincide with the nodal tractions $\mathbf{f}$, i.e.,

$$
\mathbf{S}_{\omega} \mathbf{u}=\mathbf{f}
$$

with $\mathbf{S}_{\omega}=\mathbf{S}(\chi)$, where Equations (14)-(16) are evaluated for $\chi=(\mathrm{i} \omega)^{2}$. Note that the size of $\mathbf{S}_{\boldsymbol{\omega}}$ corresponds with the number of degrees on the boundary.

In the time domain, the continued-fraction approach can be resolved by introducing auxiliary variables for the displacement $\mathbf{u}^{\top}=\left(\left(\mathbf{u}^{(0)}\right)^{\top}, \ldots,\left(\mathbf{u}^{(M)}\right)^{\top}\right)$, where each $\mathbf{u}^{(i)}$ is associated with one continuedfraction step. Equations (14)-(16) can be re-written and transferred to the time domain [13]

$$
(\mathrm{i} \omega)^{2} \mathbf{M}_{t} \mathbf{u}=-\mathbf{K}_{t} \mathbf{u}+\mathbf{f}_{t} \rightsquigarrow \partial_{t t} \mathbf{M}_{t} \mathbf{u}=-\mathbf{K}_{t} \mathbf{u}+\mathbf{f}_{t}
$$


with

$$
\begin{aligned}
\mathbf{M}_{t} & =\left(\begin{array}{ccccc}
\mathbf{M} & -\mathbf{X}^{(1)} & \mathbf{0} & \ldots & \mathbf{0} \\
\left(-\mathbf{X}^{(1)}\right)^{\top} & \mathbf{S}_{1}^{(1)} & -\mathbf{X}^{(2)} & \ddots & \vdots \\
\mathbf{0} & \left(-\mathbf{X}^{(2)}\right)^{\top} & \ddots & \ddots & \mathbf{0} \\
\vdots & \ddots & \ddots & \ddots & -\mathbf{X}^{(M)} \\
\mathbf{0} & \ldots & \mathbf{0} & \left(-\mathbf{X}^{(M)}\right)^{\top} & \mathbf{S}_{1}^{(M)}
\end{array}\right), \quad \mathbf{f}_{t}=\left(\begin{array}{c}
\mathbf{f}^{(0)} \\
\mathbf{f}^{(1)} \\
\vdots \\
\vdots \\
\mathbf{f}^{(M)}
\end{array}\right), \\
\mathbf{K}_{t} & =\operatorname{diag}\left(\mathbf{K}, \mathbf{S}_{0}^{(1)}, \ldots, \mathbf{S}_{0}^{(M)}\right),
\end{aligned}
$$

where $\mathbf{M}_{t}$ is the mass matrix, $\mathbf{K}_{t}$ is the static stiffness matrix, and $\mathbf{f}_{t}$ the time-dependent traction vector. The matrices $\mathbf{M}_{t}, \mathbf{K}_{t}$ are of size $n \times n$, where $n$ is the number of degrees on the boundary times $M+1$. For the following investigations, we choose $M=\mathrm{p}$, where $\mathrm{p}$ is the polynomial degree of the shape functions. To solve Equation (18), many time-stepping methods can be employed. Here, the implicit Newmark-beta method with $\beta=0.25$ and $\gamma=0.5$ is used with the displacement as the primary variable, leading to the dynamic stiffness matrix

$$
\mathbf{S}_{t}=\mathbf{K}+\frac{1}{\beta(\Delta t)^{2}} \mathbf{M}
$$

The continuity condition is enforced weakly in the Mortar Method. This is done by adding Lagrange multiplier terms (Equation (22)) in the weak form, see Equation (7).
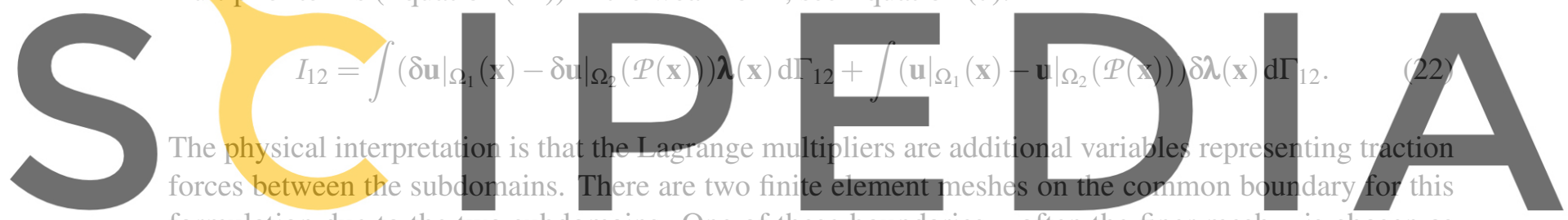

formulation due to the two subdomains. One of these boundaries - often the finer mesh - is chosen as

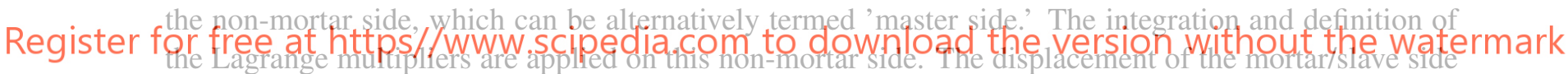
is evaluated at the nearest point projection $\mathcal{P}$. For interpolating the Lagrange multipliers, spectral shape functions of the same degree as for the displacements are used in nearly all elements. The Lagrange multipliers are continuous on each internal boundary. The exceptions are elements adjacent to a cross-point, at which at least three subdomains intersect. See Figure 7a) for an example. The Lagrange multipliers are discontinuous across at this cross-point for all polynomial degrees. In general, the cross-point of a domain can be over-constrained by the Mortar Method [1, 23]. To counteract the over-constraining, a cross-point modification can be applied, for example, the polynomial degree of elements next to a crosspoint can be lowered by one [1]. For linear shape functions, this leads to discontinuous, constant shape functions adjacent to a cross-point.

An exact integration is applied to reach higher-order convergence. The process is shown in Figure $2 \mathrm{~b}$ ), where both sides are separated by a small gap. The quadrature points of a 3-point Gaussian integration are marked by ' $\diamond$ ' on the non-mortar side and ' $x$ ' on the mortar side, respectively. 
The final form of the global stiffness matrix constitutes a saddle point problem

$$
\left(\begin{array}{ccc}
\mathbf{S}_{1} & \mathbf{0} & \mathbf{L}_{1} \\
\mathbf{0} & \mathbf{S}_{2} & \mathbf{L}_{2} \\
\mathbf{L}_{1}^{\top} & \mathbf{L}_{2}^{\top} & \mathbf{0}
\end{array}\right)\left(\begin{array}{l}
\mathbf{u}_{1} \\
\mathbf{u}_{2} \\
\boldsymbol{\lambda}
\end{array}\right)=\left(\begin{array}{c}
\mathbf{f}_{1} \\
\mathbf{f}_{2} \\
\mathbf{0}
\end{array}\right),
$$

where $\mathbf{S}_{i}$ is the dynamic stiffness matrix of each subdomain - Equation (17) for the frequency domain and Equation (21) for the time domain. $\mathbf{L}_{i}$ are the matrices associated with Equation (22) coupling the Lagrange multiplier vector and the displacement of each subdomain.

The saddle point problem quite naturally leads to a parallelization process by considering the first step of a Uzawa-like-algorithm [22] which solves Equation (23) equivalently

$$
\begin{aligned}
\mathbf{S}_{i} \mathbf{y}_{i} & =\mathbf{f}_{i} & & \forall i \in\{1,2\}, \\
\mathbf{C} \boldsymbol{\lambda} & =\sum_{i} \mathbf{L}_{i} \mathbf{y}_{i} & & \mathbf{C}=\sum_{i} \mathbf{L}_{i}^{\top} \mathbf{S}_{i} \mathbf{L}_{i}, \\
\mathrm{~S}_{i} \mathbf{u}_{i} & =\mathbb{f}_{i}-\mathbb{L}_{i} \lambda & & \forall i \in\{1,2\},
\end{aligned}
$$

where the first and last equation can be solved in parallel, and the second equation transfers information from one subdomain to the other. By the numerical examples in the next section, we show that the Schur complement matrix $\mathbf{C}$ is stable in the context of the SBFEM. In this preliminary study, a direct solver is applied to each matrix system. For the time domain, the Cholesky factorization is used as a pre-conditioner for $\mathbf{S}_{i}$ as well as the Schur complement matrix $\mathbf{C}$.
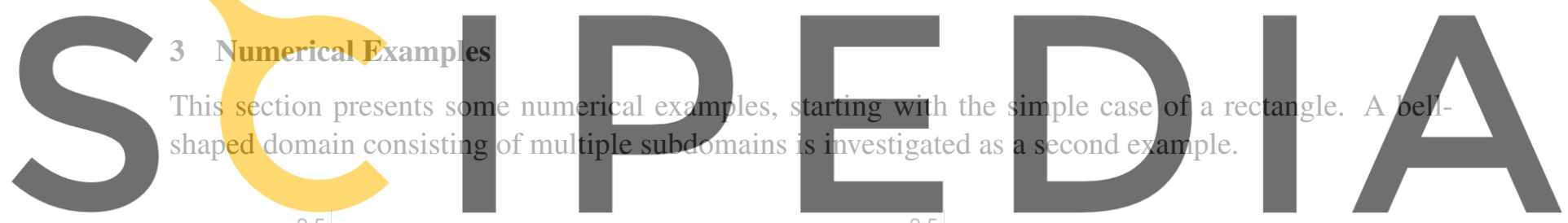

Register for free at htps//wwwscipedia.com to down̂loga the version without the watermark

a)

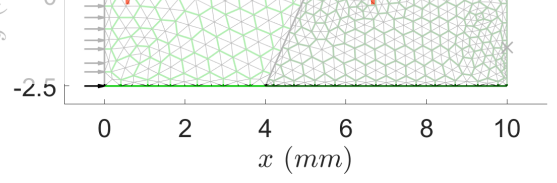

Figure 3: Problem overview for the rectangle: a) SBFEM-mesh b) Spectral element mesh

Figure 3a) shows a rectangular domain which is split into two subdomains with non-matching meshes. This domain is subject to a uniform normal traction on the left side. The traction is indicated by black arrows in the same figure. We will show results for the time and frequency domain, starting with the former. The traction varies in time as a Gaussian pulse with a center frequency $f_{0}$ of $1 \mathrm{MHz}$, i.e.,

$$
\tau(t)=\sin \left(2 \pi t f_{0}\right) \cdot \exp \left(-0.5\left(t-0.25 f_{0}^{-1}\right)^{2} /\left(f_{0}^{-1}\right)^{2}\right) .
$$

The material parameters are given in Table 1 and plane strain is assumed. The time step is chosen as $\left(100 f_{0}\right)^{-1}$ in the Newmark method. The SBFEM and the reference solution use the same Newmark method with the same time resolution, hence, the error will be dominated by the spatial discretization. 
Figure 4a) shows the result of $x$-displacement at the upper black point marked in Figure 3a). The SBFEMapproximation is compared to a reference solution calculated by means of a very highly p-refined Spectral Element approximation (SEM) - also shown in Figure 4a). We observe a good agreement between both methods. For a deeper investigation, the error between the reference solution and the SBFEM is computed as follows

$$
\text { error }=\sqrt{\sum_{i} \sum_{j}\left(u^{h}\left(P_{j}, t_{i}\right)-u^{\mathrm{ref}}\left(P_{j}, t_{i}\right)\right)^{2}} / \sqrt{\sum_{i} \sum_{j}\left(u^{\mathrm{ref}}\left(P_{j}, t_{i}\right)\right)^{2}},
$$

where $P_{i}$ are the two Gaussian integration points on the right boundary (marked with black crosses). Figure 5 shows the error for h-refinement and p-refinement, respectively. The h-refinement is based

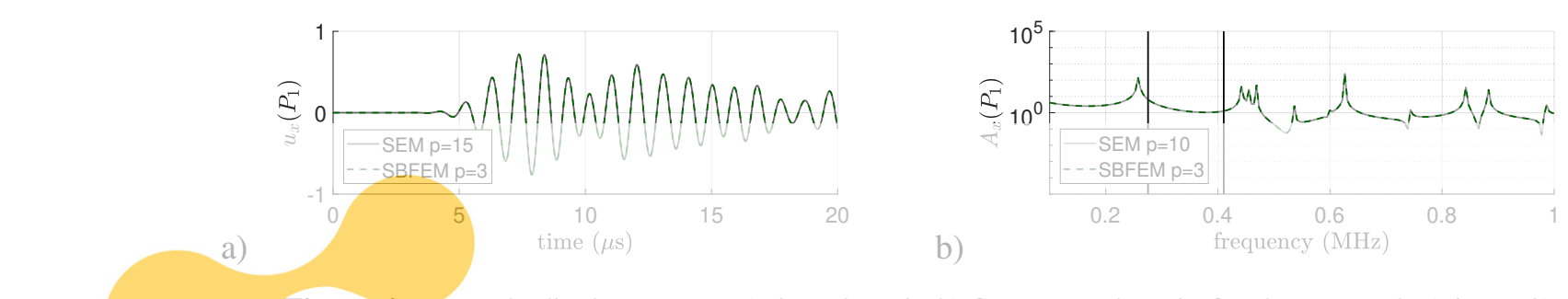

Figure 4: Example displacements: a) time domain b) frequency domain for the rectangle (Figure 3)

on a new triangulation for each refinement step. For all figures, 'DoF' is an abbreviation for all the degrees of freedom, incitin rates since the plots are However, comparing the we observe that the lines approximation and exponential convergence for $\mathrm{p}$ optimal rates of convergence, so Figure 5 indicate:
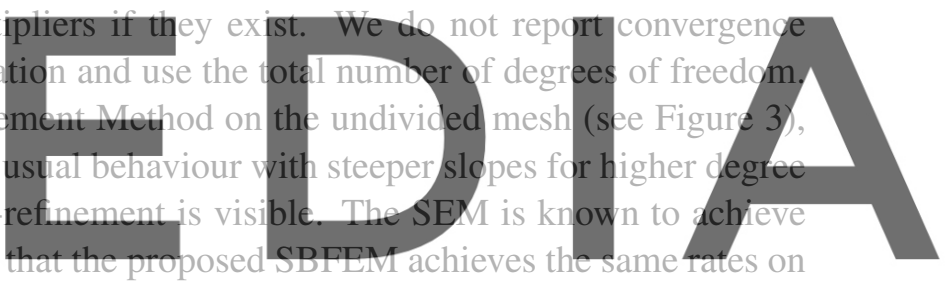
subdivided meshes. Note that this study only attempts to demonstrate the validity of the approach while

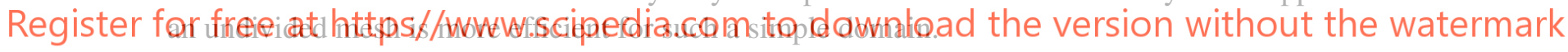

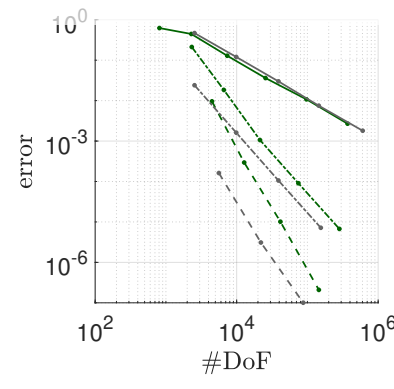

a)

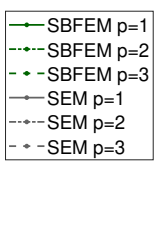

Figure 5: Error time domain: a) h-refinement b) p-refinement for the rectangle (Figure 3)

For the simulation in the frequency domain, unit tractions for 200 frequencies uniformly spaced between $0 \mathrm{MHz}$ and $1 \mathrm{MHz}$ are used. Figure $4 \mathrm{~b}$ ) shows the results for the SBFEM on two subdomains and 
the Spectral Element Method reference solution on the undivided domain. The error for both methods between the displacement evaluated at the same locations as in the time domain is summed in the range marked by the two black lines in Figure 4b). This range was chosen to avoid the resonance peaks where the numerical solution tends to be inaccurate. The error is shown in Figure 6 for h-and p-refinement. In the case of h-refinement, the same sequence of polygonal meshes is used as for the time domain. The number of degrees of freedom is much lower because only degrees on the boundary are required in the frequency domain.
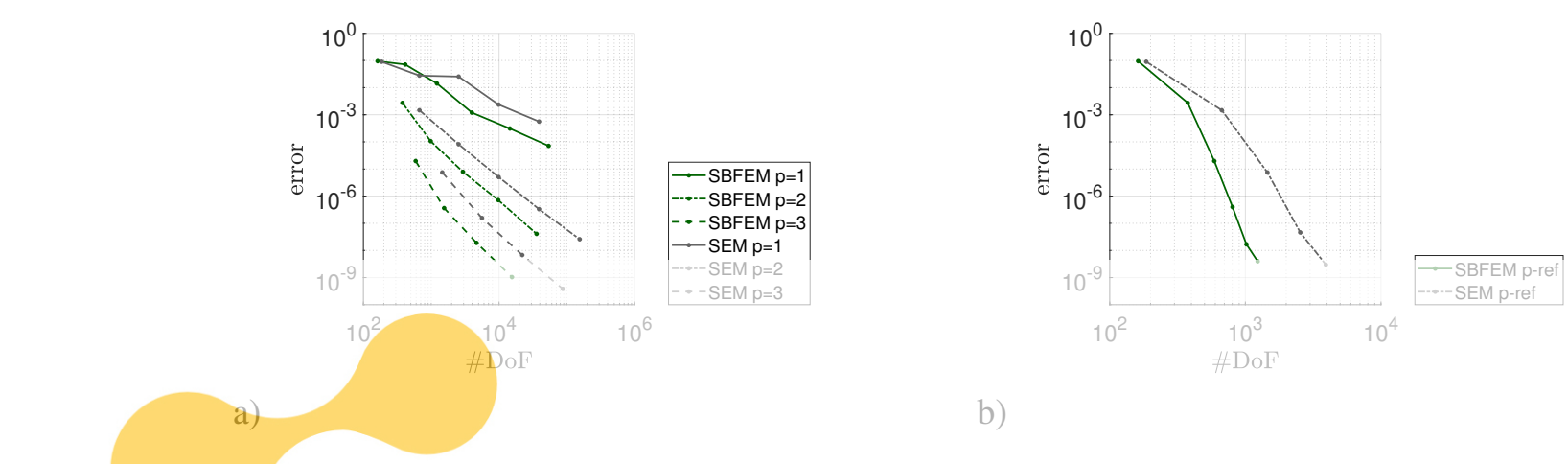

Figure 6: Error frequency domain: a) h-refinement b) p-refinement for the rectangle (Figure 3)

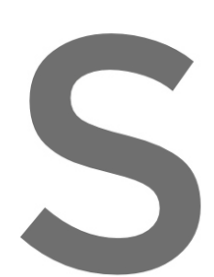

The final example show

at the top surface. The

$2 \mathrm{MHz}$. The material prop

the error for h-refinement

at the points marked with black

is applied. However, i

behaviour.

Register for free at https//www.scipedia.com to download the version without the watermark
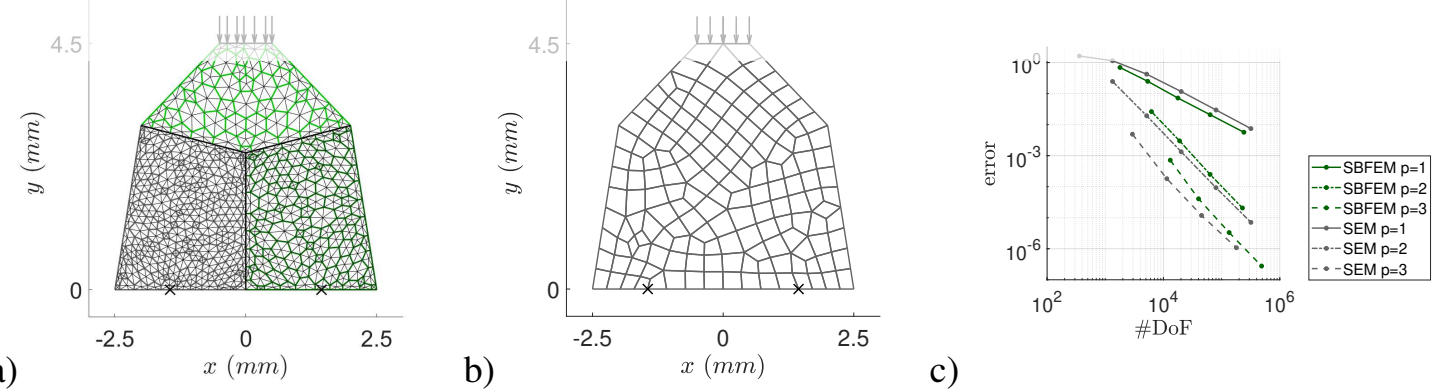

c)

Figure 7: Bell-shaped domain: a) SBFEM-mesh b) Spectral element mesh c) Error for h-refinement

\section{Conclusions}

The extension of the Scaled Boundary Finite Element Method by the Mortar Method and domain decomposition is straightforward. The formulation is stable for higher-order shape functions in both the 
Table 1: Structural steel

\begin{tabular}{lll|ll|lll}
\multicolumn{8}{c}{ Isotropic material } \\
\hline$E:$ & 200 & $\mathrm{GPa}$ & $\mathrm{v}:$ & 0.3 & $\rho:$ & 7.85 & $\mathrm{~g} \mathrm{~cm}^{-3}$
\end{tabular}

time- and the frequency-domain. The usual convergence behavior is observed if the shape functions used for the displacement and the Lagrange multipliers are of the same degree (- except elements adjacent to a cross-point). A cross-point modification is applied to counteract an over-constraining of the approximation if several subdomains intersect. The results are improved by an exact quadrature for the interface. For these small-scale examples, no decrease in the computation time was achieved due to the parallelization.

\section{Acknowledgements}

The authors thankfully acknowledge financial support from the German Research Foundation (Deutsche Forschungsgemeinschaft (DFG) - project: 428590437).

\section{REFERENCES}

[1] Wohlmuth, B. I. "A mortar finite element method using dual spaces for the Lagrange multiplier." SIAM Journal on Numerical Nnalysis 38.3 (2000): 989-1012.

[2] Seshaiyer, P., and Manil S. "Convergence results for non-conforming hp methods: The mortar finite

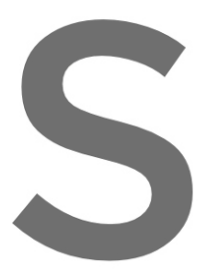
element method."

[3] Casadei, F., Gabellini, E., Foria ement method for Mechanics and En

[4] Imperiale, A., and D
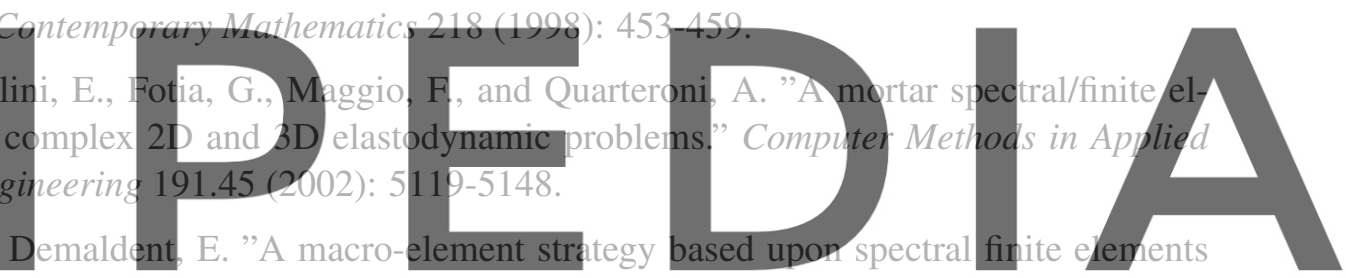

and mortar elements for transient wave propagation modeling. Application to ultrasonic testing

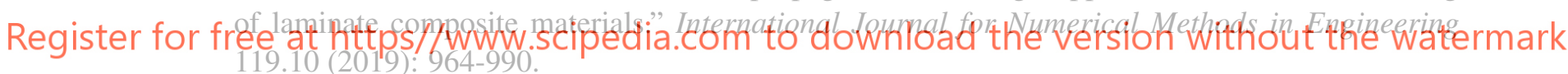

[5] Hauret, P., and Le Tallec, P. "A discontinuous stabilized mortar method for general 3d elastic problems." Computer Methods in Applied Mechanics and Engineering 196.49-52 (2007): 4881-4900.

[6] Mengolini, M., Matías F. B. and Alejandro M. A. "An engineering perspective to the virtual element method and its interplay with the standard finite element method." Computer Methods in Applied Mechanics and Engineering 350 (2019): 995-1023.

[7] Zhang, P., Du, C., Tian, X., and Jiang, S. "A scaled boundary finite element method for modelling crack face contact problems." Computer Methods in Applied Mechanics and Engineering 328 (2018): 431-451.

[8] Ghosh, S., and Mallett, R. L. "Voronoi cell finite elements." Computers \& Structures 50.1 (1994): 33-46.

[9] Da Veiga, L. B., Brezzi, F., and Marini, L. D. "Virtual elements for linear elasticity problems." SIAM Journal on Numerical Analysis 51.2 (2013): 794-812. 
[10] Floater, M. S., and Lai, M. J. "Polygonal spline spaces and the numerical solution of the Poisson equation." SIAM Journal on Numerical Analysis 54.2 (2016): 797-824.

[11] Sukumar, N., and Tabarraei, A. "Conforming polygonal finite elements." International Journal for Numerical Methods in Engineering 61.12 (2004): 2045-2066.

[12] Sukumar, N. "Quadratic maximum-entropy serendipity shape functions for arbitrary planar polygons." Computer Methods in Applied Mechanics and Engineering 263 (2013): 27-41.

[13] Bazyar, M. H., and Song, C. "A continued-fraction-based high-order transmitting boundary for wave propagation in unbounded domains of arbitrary geometry." International Journal for Numerical Methods in Engineering 74.2 (2008): 209-237.

[14] Chen, D., Birk, C., Song, C., and Du, C. "A high-order approach for modelling transient wave propagation problems using the scaled boundary finite element method." International Journal for Numerical Methods in Engineering 97.13 (2014): 937-959.

[15] Gravenkamp, H., and Natarajan, S. "Scaled boundary polygons for linear elastodynamics." Computer Methods in Applied Mechanics and Engineering 333 (2018): 238-256.

[16] Bulling, J., Gravenkamp, H., and Birk, C. "A high-order finite element technique with automatic treatment of stress singularities by semi-analytical enrichment." Computer Methods in Applied Mechanics and Engineering 355 (2019): 135-156.

[17] Gravenkamp, H. "Efficient simulation of elastic guided waves interacting with notches, adhesive joints, delaminations and incined edges in plate structures." Utrasonics \&2 (2018) 101-113.
Lugovtsova, Y., Bulling, J., Boller, C., and Prager, J. "Analysis of guided wave propagation in
a multi-layered structure in view of structural health monitoring." Applied Sciences 9.21 (2019):
4600.
Geuzaine, C., and Remacle., J F. "Gmsh: A 3-D finite element mesh generator with built-in pre-

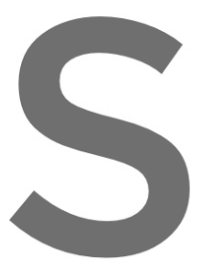

[18] L and post-processing facilities." International Journal for Numerical Methods in Engineering 79.11

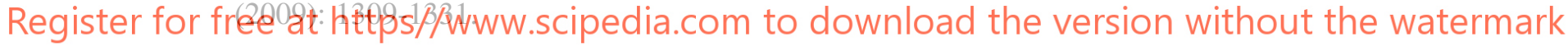

[20] Ooi, E. T., Song, C., Tin-Loi, F., and Yang, Z. "Polygon scaled boundary finite elements for crack propagation modelling." International Journal for Numerical Methods in Engineering 91.3 (2012): 319-342.

[21] Gravenkamp, H., Saputra, A. A., and Duczek, S. "High-order shape functions in the scaled boundary finite element method revisited." Archives of Computational Methods in Engineering (2019): $1-22$.

[22] Bramble, J. H., Pasciak, J. E., and Vassilev, A. T. "Analysis of the inexact Uzawa algorithm for saddle point problems." SIAM Journal on Numerical Analysis 34.3 (1997): 1072-1092.

[23] Brivadis, E., Buffa, A., Wohlmuth, B., and Wunderlich, L. ’Isogeometric mortar methods." Computer Methods in Applied Mechanics and Engineering 284 (2015): 292-319. 